\title{
A Downmodulated MicroRNA Profiling in Patients with Gastric Cancer
}

\author{
Tao Zhang, Chang Liu, Shi Huang, Yuanping Ma, Jiansong Fang, and Yuanneng Chen \\ Department of Gastroenterology, Ruikang Hospital, Guangxi Traditional Chinese Medical University, Nanning, \\ Guangxi 530011, China \\ Correspondence should be addressed to Yuanneng Chen; cyn60668@aliyun.com
}

Received 10 December 2016; Revised 24 February 2017; Accepted 9 March 2017; Published 4 May 2017

Academic Editor: Nicola Silvestris

Copyright (c) 2017 Tao Zhang et al. This is an open access article distributed under the Creative Commons Attribution License, which permits unrestricted use, distribution, and reproduction in any medium, provided the original work is properly cited.

Objective. Here, we aim to investigate the microRNA (miR) profiling in human gastric cancer (GC). Methods. Tumoral and matched peritumoral gastric specimens were collected from 12 GC patients who underwent routine surgery. A highthroughput miR sequencing method was applied to detect the aberrantly expressed miRs in a subset of 6 paired samples. The stem-loop quantitative real-time polymerase chain reaction (qRT-PCR) assay was subsequently performed to confirm the sequencing results in the remaining 6 paired samples. The profiling results were also validated in vitro in three human GC cell lines (BGC-823, MGC-803, and GTL-16) and a normal gastric epithelial cell line (GES-1). Results. The miR sequencing approach detected 5 differentially expressed miRs, hsa-miR-132-3p, hsa-miR-155-5p, hsa-miR-19b-3p, hsa-miR204-5p, and hsa-miR-30a-3p, which were significantly downmodulated between the tumoral and peritumoral GC tissues. Most of the results were further confirmed by qRT-PCR, while no change was observed for hsa-miR-30a-3p. The in vitro finding also agreed with the results of both miR sequencing and qRT-PCR for hsa-miR-204-5p, hsa-miR-155-5p, and hsa-miR132-3p. Conclusion. Together, our findings may serve to identify new molecular alterations as well as to enrich the miR profiling in human GC.

\section{Introduction}

Gastric cancer (GC) is among the most common cancers and second leading cause of global cancer mortality [1,2]. Surgical resection still remains the most promising intervention towards localized GC tumors, whereas the advanced-stage GC patients who develop recurrent diseases exhibit extremely poor quality of life as well as survival rates. Scientists have identified various genetic alterations that affect GC tumorigenesis and progression over the past decade $[3,4]$. To date, however, genetic markers for GC tumorigenesis or progression have not been identified.

MicroRNAs (miRs), a class of small noncoding RNAs, play an important role in a variety of cellular processes, including cell development, differentiation, apoptosis, and proliferation [5-7]. miRs exert negative genetic regulation by binding to complementary sites in the 3 -untranslated regions of the targeted messenger RNAs (mRNAs) [8]. Recently, miRs have been implicated in cancer development by acting as either tumor oncogenes or suppressors [9-11]. Accumulating body of evidence shows that miRs are significantly modulated in nearly all types of tumors, including human GC [12]. Moreover, the altered levels of miRs in tumors highly correlate with progression as well as prognosis of human tumors [13-15]. Interestingly, reconstitution of tumor suppressor miRs [16], or sequence-specific knockdown of oncogenic miRs [17], has produced favorable antitumor outcomes. Therefore, miRs are possible therapeutic targets for human tumors, including GC.

In the present study, we investigated the miR profiling in a small group of Chinese GC patients by applying a high- 
throughput miR sequencing method and then validated the results by performing stem-loop qRT-PCR in vivo and in vitro.

\section{Materials and Methods}

2.1. Ethics Statement. The study was approved by the Ethics Committee of Ruikang Hospital of Guangxi Traditional Chinese Medical University (Guangxi, China). All participants were explained their participation rights and signed the written informed consent.

2.2. Study Population. We recruited 12 GC patients from the Affiliated Ruikang Hospital of Guangxi University of Chinese Medicine between 2014 and 2015. They were free of any other cancers. After routine surgery, we collected tissue samples from the tumor lesion as well as from the peritumoral mucosa, which was defined as tissue that is located at $2 \mathrm{~cm}$ from the resection margin of the tumor lesion, from each participant. The TNM staging system confirmed that all the cancer samples were at T1N0M0 stage. And we reported here that all the cancer samples were collected from the gastric antrum, with none free of Helicobacter pylori. H\&E stain indicated a $100 \%$ of gastric adenocarcinoma for the samples. In order to identify the differentially expressed miRs between the tumoral and the peritumoral samples, we first constructed miR sequencing library on 6 participants ( 3 females and 3 males, aged 52 to 71 years old) with gastric tumors and matched peritumoral tissues and then screened the differentially expressed miRs between them. Then, we validated the results on the remaining 6 patients ( 3 females and 3 males, aged 56 to 70 years old) using the stem-loop quantitative real-time polymerase chain reaction (qRT-PCR) assay.

2.3. Cell Cultures. Three human GC cell lines (BGC-823, MGC-803, and GTL-16) as well as a normal gastric epithelial cell line (GES-1) were cultured in DMEM medium (Invitrogen, Carlsbad, CA, USA) supplemented with $10 \%$ fetal bovine serum, streptomycin $(100 \mu \mathrm{g} / \mathrm{ml})$, and penicillin $(100 \mathrm{U} / \mathrm{ml})$ at $37^{\circ} \mathrm{C}$ in an incubator with $5 \% \mathrm{CO}_{2}$.

2.4. RNA Isolation and Quality Control. Total RNA was isolated using the mirVana ${ }^{\mathrm{TM}}$ miRNA Isolation Kit (Ambion, Austin, TX, USA) according to the manufacturer's protocol. Notably, the cells indicated above were washed for 3 times with phosphate-buffered saline before RNA extraction. The concentration and purity of RNA samples were determined by a NanoDrop ND-1000 spectrophotometer (Nanodrop Technologies, Wilmington, DE, USA).

2.5. High-Throughput miR Sequencing Method. We performed the high-throughput miR sequencing on 6 patients with gastric tumors and matched peritumoral tissues. The miR sequencing library was constructed by applying the Illumina TruSeq Small RNA Sample Prep Kit Set A (San Diego, CA, USA). Briefly, total RNA of each sample was used to prepare the miR sequencing library, which included the following steps: (1) 3'-adaptor ligation, (2) 5'-adaptor ligation, (3) cDNA synthesis, (4) PCR amplification, and (5) size selection of $\sim 135-155$ bp PCR-amplified fragments (corresponding to $\sim 15-35$ nt small RNAs). The libraries were denatured as single-stranded DNA molecules, captured on Illumina flow cells, amplified in situ as clusters, and finally sequenced for 36 cycles on the Illumina HiSeq ${ }^{\mathrm{TM}} 2000$ System (San Diego, CA, USA) as per the manufacturer's instructions. After sequencing, the Solexa CHASTITY quality-filtered reads were harvested as clean reads. The adaptor sequences were trimmed and the adaptor-trimmed reads ( $\geq 15 \mathrm{nt})$ were left. miRDeep2 software was used to predict the novel miRs with these trimmed reads [18]. Then, the trimmed reads were aligned to merged pre-miRNA databases (known pre-miR from miRBase v21 plus the newly predicted pre-miRs) using Novoalign software v2.07.11 (http://www.novocraft.com) with at most one mismatch. The numbers of mapped tags were defined as the raw expression levels of that miR. To correct for the difference in tag counts between samples, the tag counts were scaled to TPM (the copy number of transcripts per million) based on the total number of tag aligned [19]. Fold change and $p$ value were calculated and used to identify the differentially expressed miRs between tumoral and peritumoral tissues. The miRs, which matched $p<0.05$ and fold change $>2$ (upregulated) or $<0.5$ (downregulated), were considered differentially expressed miRs.

2.6. $q R T-P C R$ Validation. In order to further validate the results of the differentially expressed miRs identified by the high-throughput $\mathrm{miR}$ sequencing method between the tumoral and peritumoral tissues, the stem-loop qRT-PCR method using a mirVana miRNA Detection Kit and genespecific primers (Ambion) was introduced to quantify the expression levels of the miRs in vivo on the remaining 6 GC patients as well as in vitro on the cell lines indicated above [20]. The PCR reactions $(20 \mu \mathrm{l})$ were performed in 96-well plates and run in triplicate on a Bio-Rad CFX96 Touch Real-Time PCR Machine. Thermal cycling was organized in 3 repeated steps: the first denaturation step of $95^{\circ} \mathrm{C}$ for 10 minutes, followed by 40 cycles of $95^{\circ} \mathrm{C}$ for 15 seconds, and $60^{\circ} \mathrm{C}$ for 1 minute. RNU6B was used as endogenous controls to normalize the relative expression levels of the miRs.

2.7. Statistical Analyses. STATISTICA 10 (Dell, Round Rock, TX, USA) was used to perform the statistical analyses. Graph preparations were carried out by Microsoft Office Excel 2007. Student $t$-tests were applied to compare data between groups. A $p$ value less than 0.05 was considered to be statistically significant.

\section{Results}

3.1. RNA Quality Control. Because construction of miR sequencing library requires high-quality RNA samples, thus we would not consider those that failed to meet $\mathrm{OD}_{260 / 280}$ ratio $>1.8$ as well as $\mathrm{OD}_{260 / 230}$ ratio $>1.8$. We show in Table 1 that all the RNA samples used in the present study were tested qualified for the construction of miR sequencing library.

3.2. Quality Assessment of the $m i R$ Sequencing Library. Quality assessment of the sequencing library was determined by an Agilent 2100 Bioanalyzer using the Agilent 
TABLE 1: Quality control of RNA samples for the construction of miR sequencing library.

\begin{tabular}{lccc}
\hline Sample ID & OD $_{260 / 280}$ ratio & OD $_{260 / 230}$ ratio & Conc. $(\mathrm{ng} / \mu \mathrm{l})$ \\
\hline $\mathrm{T}-1$ & 1.95 & 2.25 & 1218 \\
$\mathrm{~T}-2$ & 1.92 & 2.27 & 960 \\
$\mathrm{~T}-3$ & 1.96 & 2.28 & 1850 \\
$\mathrm{~T}-4$ & 1.99 & 2.32 & 1348 \\
$\mathrm{~T}-5$ & 1.99 & 2.30 & 1671 \\
$\mathrm{~T}-6$ & 1.97 & 2.30 & 1273 \\
PT-1 & 1.88 & 2.11 & 1327 \\
PT-2 & 1.92 & 2.18 & 1590 \\
PT-3 & 1.94 & 2.26 & 1789 \\
PT-4 & 1.94 & 2.29 & 1430 \\
PT-5 & 1.81 & 1.81 & 1350 \\
PT-6 & 1.81 & 1.81 & 1106 \\
\hline
\end{tabular}

T: gastric tumor; PT: peritumoral tissue.

DNA 1000 chip kit (Santa Clara, CA, USA). We selected size of $\sim 135-155$ bp PCR-amplified fragments. The library, of which the concentration was below $1.0 \mathrm{ng} / \mu \mathrm{l}$, would not be taken into consideration. Table 2 displays that all the libraries were tested qualified.

3.3. Altered miR Expression Pattern in Human GC. First, we attempted to screen the expression of human miRs of 6 GC specimens and of their matched peritumoral tissues using the high-throughput $\mathrm{miR}$ sequencing method. This approach allowed us to identify 5 differentially expressed miRs, hsa-miR-132-3p (A, $p=0.013$ ), hsa-miR-155-5p (B, $p=0.031)$, hsa-miR-19b-3p (C, $p=0.002)$, hsa-miR-204-5p (D, $p=0.016)$, and hsa-miR-30a-3p (E, $p=0.019)$, that were significantly modulated between tumoral and peritumoral tissues (Figure 1). Furthermore, the altered miR expression showed a downregulated pattern in human GC, as confirmed by the fold changes. Interestingly, the altered miR expression pattern was consistent with the previous studies for hsa-miR-155-5p [21, 22], hsa-miR-19b-3p [23], and hsa-miR-204-5p $[15,24]$ in human GC.

In order to further confirm the miR sequencing results, we then performed stem-loop qRT-PCR on the remaining 6 GC patients for the 5 identified miRs between tumoral and peritumoral tissues. Consistent with the miR sequencing results, we observed that hsa-miR-132-3p $(t=-2.690$, $p=0.023)$, hsa-miR-155-5p $(t=-5.553, p=0.000)$, hsamiR-19b-3p $(t=-2.458, p=0.034)$, and hsa-miR-204-5p $(t=-3.843, p=0.003)$ were significantly downmodulated in human GC compared with peritumoral tissues (Figure 2). However, the expression of hsa-miR-30a-3p remained unaltered (data not shown).

As shown above, the miR sequencing and qRT-PCR results agreed with each other for the modulation pattern of hsa-miR-132-3p, hsa-miR-155-5p, hsa-miR-19b-3p, and hsa-miR-204-5p in human GC. We speculated that similar result(s) might probably be obtained in vitro as well. To achieve this, we next investigated the expression levels of the 5 indicated miRs in three human GC cell lines (BGC-823,
TABLE 2: Quality assessment of the miR sequencing library.

\begin{tabular}{lcccc}
\hline $\begin{array}{l}\text { Sample } \\
\text { ID }\end{array}$ & $\begin{array}{c}\text { Size } \\
(\mathrm{bp})\end{array}$ & $\begin{array}{c}\text { Conc. } \\
(\mathrm{ng} / \mu \mathrm{l})\end{array}$ & $\begin{array}{c}\text { Volume } \\
(\mu \mathrm{l})\end{array}$ & $\begin{array}{c}\text { Total amount } \\
(\mathrm{ng})\end{array}$ \\
\hline T-1 & 147 & 1.94 & 20 & 38.8 \\
T-2 & 149 & 1.66 & 20 & 33.2 \\
T-3 & 147 & 2.09 & 20 & 41.8 \\
T-4 & 146 & 1.11 & 20 & 22.2 \\
T-5 & 139 & 1.87 & 20 & 37.4 \\
T-6 & 147 & 1.29 & 20 & 25.8 \\
PT-1 & 148 & 1.63 & 20 & 32.6 \\
PT-2 & 147 & 1.43 & 20 & 28.6 \\
PT-3 & 146 & 1.33 & 20 & 26.6 \\
PT-4 & 145 & 1.62 & 20 & 32.4 \\
PT-5 & 146 & 1.90 & 20 & 38.0 \\
PT-6 & 145 & 1.04 & 20 & 20.8 \\
\hline
\end{tabular}

T: gastric tumor; PT: peritumoral tissue.

MGC-803, and GTL-16) and a normal gastric epithelial cell line (GES-1). Interestingly, we observed a strong difference of the expression levels of hsa-miR-204-5p in all three human GC cell lines, MGC-803 $(t=4.261, p=0.002)$, BGC-823 $(t=4.694, p=0.001)$, and GTL-16 $(t=18.544, p=0.000)$, when compared with that in the normal gastric epithelial cell line, GES-1 (Figure 3). Similar results were also observed for hsa-miR-155-5p (MGC-803: $t=20.281, p=0.000$; BGC-823: $t=5.286, p=0.006$; and GTL-16: $t=3.196, p=0.033)$ as well as for has-miR-132-3p (MGC-803: $t=7.755, p=0.001$; BGC-823: $t=4.707, p=0.009 ;$ and GTL-16: $t=3.314$, $p=0.032$ ), as shown in Figure 3. However, we failed to replicate the in vivo findings of hsa-miR-19b-3p or hasmiR-30a-3p in the present three GC cell lines (data not shown). Taken together, our data demonstrated a downregulated miR profiling in human GC tissue, which was further agreed by in vitro results for hsa-miR-204-5p, hsa-miR155-5p, and hsa-miR-132-3p.

\section{Discussion}

miRs may act as either tumor suppressors or oncogenes in cancer development [9-11]. Accumulating body of evidence highlights miRs as molecular targets whose modulation may hold therapeutic promise towards different types of tumor [25], including GC [26]. Hundreds of differentially expressed miRs have been reported between GC specimens and their matched peritumoral tissues over the past decade [12]. Downregulated miRs frequently lead to loss of tumor suppressor activity. A recent study indicated that downregulation of a large amount of miRs played a critical role in tumorigenesis independently from the type of tumor analyzed [27]. In the present study, we identified 4 significantly downregulated miRs, hsa-miR-204-5p, hsa-miR-155-5p, hsa-miR-132-3p, and hsa-miR-19b-3p, in human GC tissues by a combination of the high-throughput $\mathrm{miR}$ sequencing approach as well as subsequent qRT-PCR validation. We also observed a downmodulated level of hsa-miR-204-5p, hsamiR-155-5p, and hsa-miR-132-3p in three human GC cell 


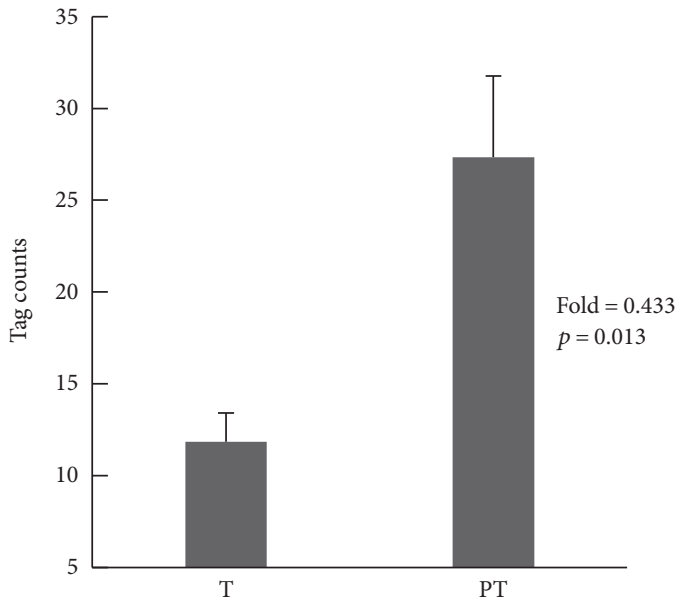

(a) hsa-miR-132-3p

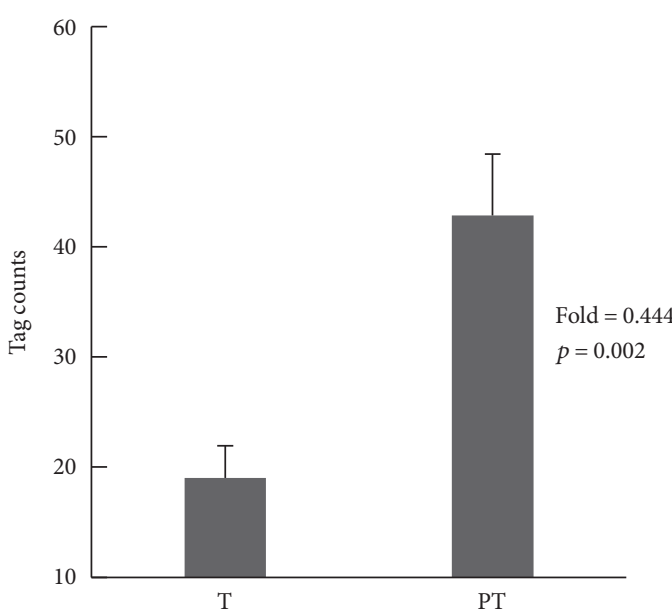

(c) hsa-miR-19b-3p

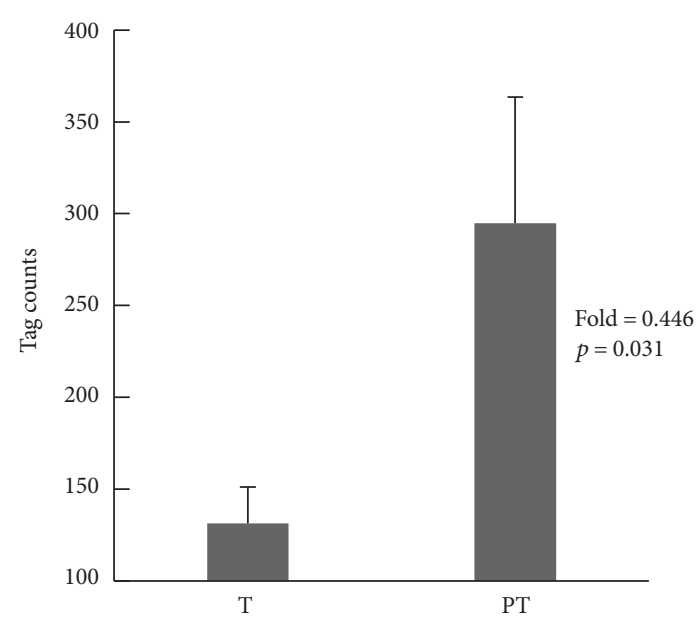

(b) hsa-miR-155-5p

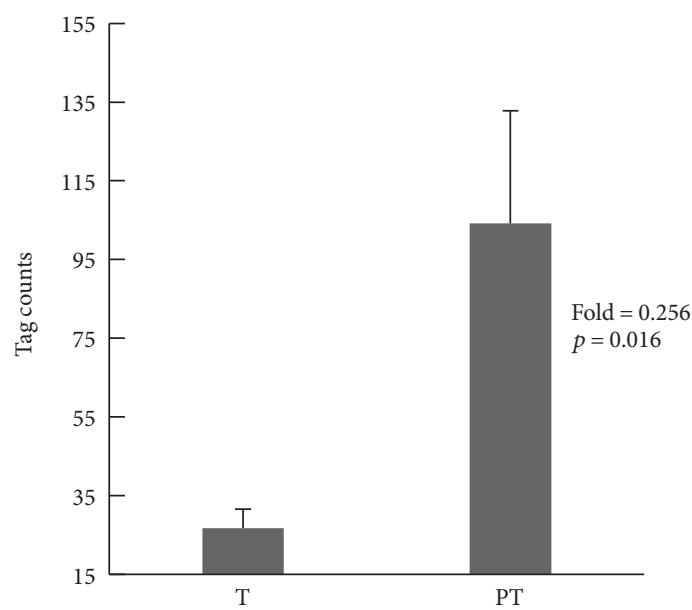

(d) hsa-miR-204-5p

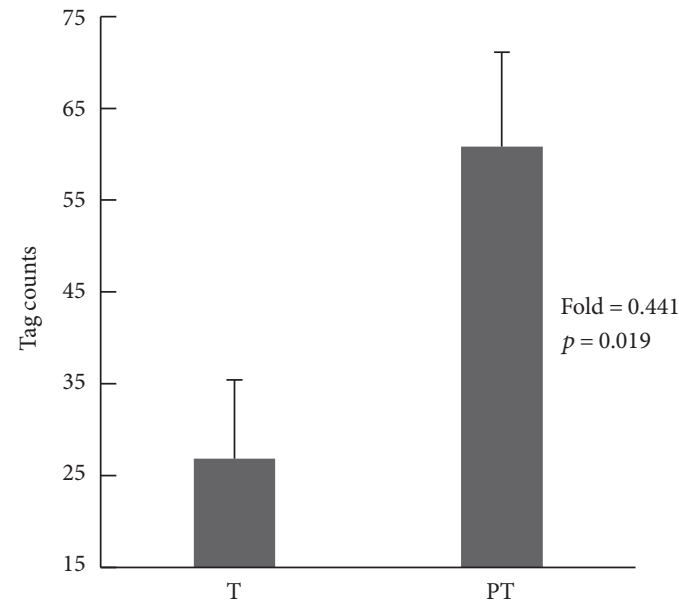

(e) hsa-miR-30a-3p

FIGURE 1: Five downregulated miRs between gastric tumor (T) and peritumoral tissue (PT) were identified by the high-throughput miR sequencing method. Tag counts refers to the normalized tag number of the mature miRs annotated in miRBase 21 (TPM), including the tag number of all samples. A fold change less than 0.5 (as well as $p$ value less than 0.05 ) indicated that the expression of the miR was downregulated in gastric tumor compared with the corresponding adjacent tissue. Data are represented as mean $\pm \mathrm{SD}, n=6$. 


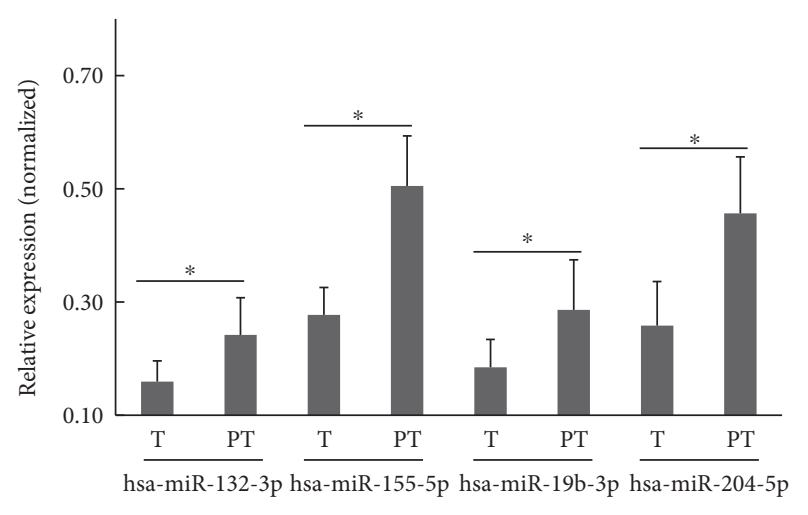

FIGURE 2: Stem-loop qRT-PCR confirmed the altered expression pattern of four miRs between tumoral $(\mathrm{T})$ and peritumoral tissues (PT) in vivo. The relative miR expression levels were normalized by RNU6B. Data are expressed as mean $\pm \mathrm{SD}, n=6$. An asterisk $\left({ }^{*}\right)$ indicates $p<0.05$.

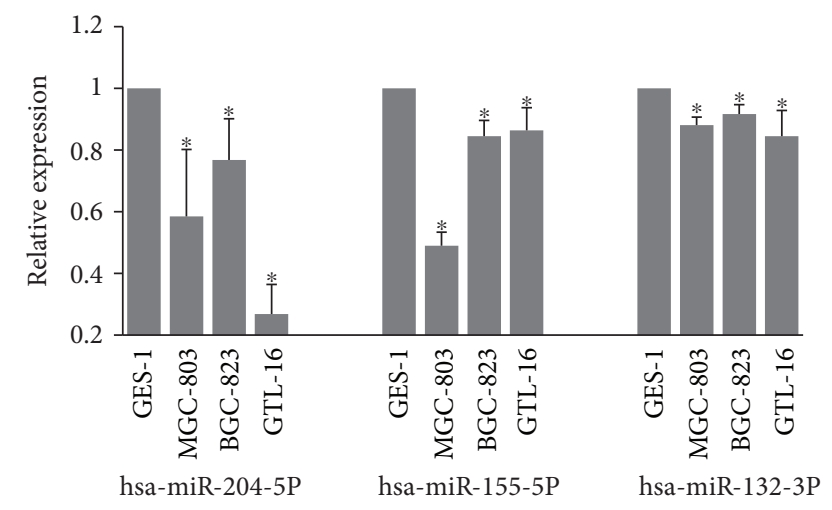

Figure 3: The expression levels of hsa-miR-204-5p, hsa-miR-155$5 p$, and hsa-miR-132-3p were relatively downregulated in three human GC cell lines. The expression levels of the three GC cell lines (MGC-803, BGC-823, and GTL-16) were calculated as relative expression to that of the control group, that is, the normal gastric epithelial cell line (GES-1). Experiments were performed in triplicate. Data are expressed as mean \pm SD. An asterisk $\left({ }^{*}\right)$ indicates $p<0.05$ (versus that in GES-1).

lines (BGC-823, MGC-803, and GTL-16) when compared with that in a normal gastric epithelial cell line (GES-1).

Accumulating studies demonstrated that the expression of miR-204 was drastically downmodulated in various types of cancer, including ovarian cancers, colorectal cancer, pediatric renal tumors, breast cancers, and glioblastoma [28-30]. In the current study, we showed a similar result in human GC as well as in several GC cell lines, which was further agreed by several other in vivo and in vitro studies [15, 24, 31]. For example, Zhang et al. demonstrated that miR-204-5p was significantly downmodulated in a GC cohort [24]. A very recent review believes that miR-204 acts as a tumor suppressor via promoting apoptosis, decreasing resistance of cancer cells to chemotherapy, and suppressing the self-renewal of cancer stem cells [32]. Interestingly, several research groups tentatively documented the underlying mechanisms that how miR-204 functions in GC development [15, 24, 31, 33].
For example, Sacconi et al. demonstrated that miR-204 targeted B cell lymphoma-2 (Bcl-2) mRNA and increased responsiveness of GC cells to 5-fluorouracil and oxaliplatin treatment, and ectopic expression of Bcl-2 counteracted miR-204 proapoptotic activity [15]. Another study evidenced that a downmodulation of miR-204 promoted GC cell invasion by activating the SIRT1- (sirtuin type 1)-LKB1 (liver kinase B1) pathway [31]. Zhang et al. identified that ubiquitinspecific peptidase 47 (USP47) and Ras-related protein Rab22A (RAB22A) were direct functional targets of miR-204-5p in GC and that miR-204-5p inhibited GC cell proliferation by downregulating USP47 and RAB22A [24]. Together, these results indicate that regulation of miR-204 expression may hold therapeutic promise for human GC.

miR-155 is primarily expressed within lymphocytes and functions as an important regulator of the immune system [34]. The underlying mechanisms have been well documented elsewhere $[35,36]$. Recent studies have shown that transgenic mice expressing miR-155 in B lymphocytes generated lymphoma, indicating that miR-155 is oncogenic $[37,38]$. This is further agreed by an accumulating body of clinical evidence showing that several types of malignancy express high level of miR-155. For instance, overexpression of miR-155 is detected in a number of B cell lymphomas [39-41]. Moreover, the oncogenic role of miR-155 is also described in several other cancers where overexpression of miR-155 frequently corresponds with poor prognosis [42-44]. However, we noticed that several miR profiling studies consistently reported a downmodulated alteration of miR-155 in GC tissues [45-47]. In the current study, we also observed similar result that miR-155 was aberrantly downmodulated in GC samples. The inconsistency of miR-155 expression pattern might be explained by the differences of tumor types and/or immune functions. More studies need to be taken to further address the biological functions of the miR in tumorigenesis.

Recent in vivo and in vitro investigations evidenced that miR-132 is involved in the development of several types of cancers, including breast cancer [48], ovarian cancer [49], lung cancer [50], and colorectal cancer [51]. Consistently, these studies agreed that the alteration of miR-132 showed a downregulated pattern. Interestingly, we also detected a similar result in GC tissues in the present study. Together with our study, these results indicate that miR-132 might act as a tumor suppressor. Moreover, several research groups tentatively explored the underlying mechanisms of miR132 in cancer development. For example, Tian et al. revealed in vitro that miR-132 inhibited cell proliferation, invasion, and migration in ovarian cancer by targeting the transcription factor E2F5 [49]. You et al. showed that miR-132 suppressed the migration and invasion of human non-small-cell lung cancer cells via targeting the EMT- (epithelial-to-mesenchymal transition-) related transcription factor ZEB2 [50]. Similar mechanism was also reported elsewhere that miR-132 inhibited colorectal cancer cell invasion and metastasis via directly targeting ZEB2 [51]. However, little is known about the underlying mechanisms that how miR-132 contributes to GC development, which calls for future investigations. 
miR-19b is fully evidenced in literature as a tumor oncogene in various types of cancers, and the underlying mechanisms are investigated accordingly [52-55]. It is also considered a novel potential biomarker to indicate progression of GC [23]. However, we failed to observe a similar result in GC tissue with a small Chinese population or in vitro with several GC cell lines in the present study. Instead, our data showed a downmodulated alteration in vivo, which indicates that miR-19b might act as a tumor suppressor towards GC. We, for the present, are unable to explain the inconsistency. A further study needs to be taken to revalidate the miR-19b expression pattern in GC.

The study has an obvious limitation, which is attributed to the small sample size. Besides, among different miR profiling studies on GC (as well as on other cancers), a certain variability of the profiling results exists and these discrepancies could be probably related to some factors, for example, test sample size and biological and analytical differences. Thus, findings and/or conclusions of the present study should be considered preliminary until replicated studies with larger sample sizes are conducted in the future.

Taken together, we in vivo and in vitro detected a set of downmodulated miRs in human GC, most of which were consistent with a plenty of previous studies investigating other types of cancer. Our results may contribute to the identification of new molecular alterations as well as to the enrichment of the miR profiling in human GC.

\section{Conflicts of Interest}

The authors declare that they have no competing interest.

\section{Authors' Contributions}

Tao Zhang analyzed the data and wrote the paper; Chang Liu, Shi Huang, Yuanping Ma, and Jiansong Fang collected the specimens, performed the experiments, and collected the data; and Yuanneng Chen designed the study and revised the manuscript. All authors have read and approved the final version to be submitted.

\section{Acknowledgments}

The present work was supported by the National Natural Science Foundation of China (no. 81360531). The authors would like to thank Guo-Kuan Chen, Shanghai KangChen Bio-tech, for the technical assistance in the miR sequencing assay.

\section{References}

[1] A. Jemal, F. Bray, M. M. Center, J. Ferlay, E. Ward, and D. Forman, "Global cancer statistics," CA: A Cancer Journal for Clinicians, vol. 61, no. 2, pp. 69-90, 2011.

[2] H. Isomoto, S. Shikuwa, N. Yamaguchi et al., "Endoscopic submucosal dissection for early gastric cancer: a large-scale feasibility study," Gut, vol. 58, no. 3, pp. 331-336, 2009.

[3] N. Jinawath, Y. Furukawa, S. Hasegawa et al., "Comparison of gene-expression profiles between diffuse- and intestinal-type gastric cancers using a genome-wide cDNA microarray," Oncogene, vol. 23, no. 40, pp. 6830-6844, 2004.
[4] M. Stock and F. Otto, "Gene deregulation in gastric cancer," Gene, vol. 360, no. 1, pp. 1-19, 2005.

[5] M. Lagos-Quintana, R. Rauhut, W. Lendeckel, and T. Tuschl, "Identification of novel genes coding for small expressed RNAs," Science, vol. 294, no. 5543, pp. 853-858, 2001.

[6] D. P. Bartel, "MicroRNAs: genomics, biogenesis, mechanism, and function," Cell, vol. 116, no. 2, pp. 281-297, 2004.

[7] V. Ambros, "The functions of animal microRNAs," Nature, vol. 431, no. 7006, pp. 350-355, 2004.

[8] L. He and G. J. Hannon, "MicroRNAs: small RNAs with a big role in gene regulation," Nature Reviews. Genetics, vol. 5, no. 7, pp. 522-531, 2004.

[9] G. Di Leva, M. Garofalo, and C. M. Croce, "MicroRNAs in cancer," Annual Review of Pathology, vol. 9, no. 1, pp. 287-314, 2014.

[10] A. Esquela-Kerscher and F. J. Slack, "Oncomirs - microRNAs with a role in cancer," Nature Reviews. Cancer, vol. 6, no. 4, pp. 259-269, 2006.

[11] G. A. Calin and C. M. Croce, "MicroRNA signatures in human cancers," Nature Reviews Cancer, vol. 6, no. 11, pp. 857-866, 2006.

[12] S. Shrestha, S. D. Hsu, W. Y. Huang et al., "A systematic review of microRNA expression profiling studies in human gastric cancer," Cancer Medicine, vol. 3, no. 4, pp. 878-888, 2014.

[13] A. J. Schetter, S. Y. Leung, J. J. Sohn et al., "MicroRNA expression profiles associated with prognosis and therapeutic outcome in colon adenocarcinoma," Journal of the American Medical Association, vol. 299, no. 4, pp. 425-436, 2008.

[14] M. Bloomston, W. L. Frankel, F. Petrocca et al., "MicroRNA expression patterns to differentiate pancreatic adenocarcinoma from normal pancreas and chronic pancreatitis," Journal of the American Medical Association, vol. 297, no. 17, pp. 1901-1908, 2007.

[15] A. Sacconi, F. Biagioni, V. Canu et al., "miR-204 targets Bcl-2 expression and enhances responsiveness of gastric cancer," Cell Death \& Disease, vol. 3, no. 11, p. e423, 2012.

[16] N. Nadiminty, R. Tummala, W. Lou et al., "MicroRNA let-7c suppresses androgen receptor expression and activity via regulation of Myc expression in prostate cancer cells," The Journal of Biological Chemistry, vol. 287, no. 2, pp. 1527-1537, 2012.

[17] J. Krutzfeldt, N. Rajewsky, R. Braich et al., "Silencing of microRNAs in vivo with 'antagomirs'," Nature, vol. 438, no. 7068, pp. 685-689, 2005.

[18] M. R. Friedlander, S. D. Mackowiak, N. Li, W. Chen, and N. Rajewsky, "miRDeep2 accurately identifies known and hundreds of novel microRNA genes in seven animal clades," Nucleic Acids Research, vol. 40, no. 1, pp. 37-52, 2012.

[19] C. Vaz, H. M. Ahmad, P. Sharma et al., "Analysis of microRNA transcriptome by deep sequencing of small RNA libraries of peripheral blood," BMC Genomics, vol. 11, no. 1, p. 288, 2010.

[20] C. Chen, D. A. Ridzon, A. J. Broomer et al., "Real-time quantification of microRNAs by stem-loop RT-PCR," Nucleic Acids Research, vol. 33, no. 20, article e179, 2005.

[21] H. Li, S. Xie, M. Liu et al., "The clinical significance of downregulation of mir-124-3p, mir-146a-5p, mir-155-5p and mir$335-5 p$ in gastric cancer tumorigenesis," International Journal of Oncology, vol. 45, no. 1, pp. 197-208, 2014.

[22] M. Zhu, M. Wang, F. Yang et al., "miR-155-5p inhibition promotes the transition of bone marrow mesenchymal stem cells to gastric cancer tissue derived MSC-like cells via NF- 
kappaB p65 activation," Oncotarget, vol. 7, no. 13, pp. 16567-16580, 2016.

[23] J. Zhang, Y. Song, C. Zhang et al., "Circulating MiR-16-5p and MiR-19b-3p as two novel potential biomarkers to indicate progression of gastric cancer," Theranostics, vol. 5, no. 7, pp. 733-745, 2015.

[24] B. Zhang, Y. Yin, Y. Hu et al., "MicroRNA-204-5p inhibits gastric cancer cell proliferation by downregulating USP47 and RAB22A," Medical Oncology, vol. 32, no. 1, p. 331, 2015.

[25] Z. Liu, A. Sall, and D. Yang, "MicroRNA: an emerging therapeutic target and intervention tool," International Journal of Molecular Sciences, vol. 9, no. 6, pp. 978-999, 2008.

[26] T. Ueda, S. Volinia, H. Okumura et al., "Relation between microRNA expression and progression and prognosis of gastric cancer: a microRNA expression analysis," The Lancet Oncology, vol. 11, no. 2, pp. 136-146, 2010.

[27] M. Ozen, C. J. Creighton, M. Ozdemir, and M. Ittmann, "Widespread deregulation of microRNA expression in human prostate cancer," Oncogene, vol. 27, no. 12, pp. 17881793, 2008.

[28] J. S. Imam, J. R. Plyler, H. Bansal et al., "Genomic loss of tumor suppressor miRNA-204 promotes cancer cell migration and invasion by activating AKT/mTOR/Racl signaling and actin reorganization," PLoS One, vol. 7, no. 12, article e52397, 2012.

[29] S. Song, A. Fajol, X. Tu, B. Ren, and S. Shi, "miR-204 suppresses the development and progression of human glioblastoma by targeting ATF2," Oncotarget, vol. 7, no. 43, pp. 70058-70065, 2016.

[30] Y. Yin, B. Zhang, W. Wang et al., "miR-204-5p inhibits proliferation and invasion and enhances chemotherapeutic sensitivity of colorectal cancer cells by downregulating RAB22A," Clinical Cancer Research, vol. 20, no. 23, pp. 6187-6199, 2014.

[31] L. Zhang, X. Wang, and P. Chen, "MiR-204 down regulates SIRT1 and reverts SIRT1-induced epithelial-mesenchymal transition, anoikis resistance and invasion in gastric cancer cells," BMC Cancer, vol. 13, no. 1, p. 290, 2013.

[32] T. Li, H. Pan, and R. Li, "The dual regulatory role of miR-204 in cancer," Tumour Biology, vol. 37, no. 9, pp. 11667$11677,2016$.

[33] Z. Liu, J. Long, R. Du, C. Ge, K. Guo, and Y. Xu, "miR-204 regulates the EMT by targeting snail to suppress the invasion and migration of gastric cancer," Tumour Biology, vol. 37, no. 6, pp. 8327-8335, 2016.

[34] E. Vigorito, S. Kohlhaas, D. Lu, and R. Leyland, "miR-155: an ancient regulator of the immune system," Immunological Reviews, vol. 253, no. 1, pp. 146-157, 2013.

[35] R. Mashima, "Physiological roles of miR-155," Immunology, vol. 145, no. 3, pp. 323-333, 2015.

[36] N. Seddiki, V. Brezar, N. Ruffin, Y. Levy, and S. Swaminathan, "Role of miR-155 in the regulation of lymphocyte immune function and disease," Immunology, vol. 142, no. 1, pp. 32$38,2014$.

[37] S. Costinean, S. K. Sandhu, I. M. Pedersen et al., "Src homology 2 domain-containing inositol-5-phosphatase and CCAAT enhancer-binding protein beta are targeted by miR-155 in B cells of Emicro-MiR-155 transgenic mice," Blood, vol. 114, no. 7, pp. 1374-1382, 2009.

[38] I. A. Babar, C. J. Cheng, C. J. Booth et al., "Nanoparticle-based therapy in an in vivo microRNA-155 (miR-155)-dependent mouse model of lymphoma," Proceedings of the National
Academy of Sciences of the United States of America, vol. 109, no. 26, pp. E1695-E1704, 2012.

[39] P. S. Eis, W. Tam, L. Sun et al., "Accumulation of miR-155 and BIC RNA in human B cell lymphomas," Proceedings of the National Academy of Sciences of the United States of America, vol. 102, no. 10, pp. 3627-3632, 2005.

[40] J. Kluiver, S. Poppema, D. de Jong et al., "BIC and miR-155 are highly expressed in Hodgkin, primary mediastinal and diffuse large B cell lymphomas," The Journal of Pathology, vol. 207, no. 2, pp. 243-249, 2005.

[41] M. Metzler, M. Wilda, K. Busch, S. Viehmann, and A. Borkhardt, "High expression of precursor microRNA-155/ BIC RNA in children with Burkitt lymphoma," Genes, Chromosomes \& Cancer, vol. 39, no. 2, pp. 167-169, 2004.

[42] M. Gironella, M. Seux, M. J. Xie et al., "Tumor protein 53induced nuclear protein 1 expression is repressed by miR-155, and its restoration inhibits pancreatic tumor development," Proceedings of the National Academy of Sciences of the United States of America, vol. 104, no. 41, pp. 16170-16175, 2007.

[43] S. Volinia, G. A. Calin, C. G. Liu et al., "A microRNA expression signature of human solid tumors defines cancer gene targets," Proceedings of the National Academy of Sciences of the United States of America, vol. 103, no. 7, pp. 2257-2261, 2006.

[44] M. F. Czyzyk-Krzeska and X. Zhang, "MiR-155 at the heart of oncogenic pathways," Oncogene, vol. 33, no. 6, pp. 677678, 2014.

[45] C. H. Kim, H. K. Kim, R. L. Rettig et al., "miRNA signature associated with outcome of gastric cancer patients following chemotherapy," BMC Medical Genomics, vol. 4, no. 1, p. 79, 2011.

[46] X. Li, F. Luo, Q. Li et al., "Identification of new aberrantly expressed miRNAs in intestinal-type gastric cancer and its clinical significance," Oncology Reports, vol. 26, no. 6, pp. 1431-1439, 2011.

[47] Y. Yao, A. L. Suo, Z. F. Li et al., "MicroRNA profiling of human gastric cancer," Molecular Medicine Reports, vol. 2, no. 6, pp. 963-970, 2009.

[48] Z. Damavandi, S. Torkashvand, M. Vasei, B. M. Soltani, M. Tavallaei, and S. J. Mowla, "Aberrant expression of breast development-related microRNAs, miR-22, miR-132, and miR-212, in breast tumor tissues," Journal of Breast Cancer, vol. 19, no. 2, pp. 148-155, 2016.

[49] H. Tian, L. Hou, Y. M. Xiong et al., "miR-132 targeting E2F5 suppresses cell proliferation, invasion, migration in ovarian cancer cells," American Journal of Translational Research, vol. 8, no. 3, pp. 1492-1501, 2016.

[50] J. You, Y. Li, N. Fang et al., "MiR-132 suppresses the migration and invasion of lung cancer cells via targeting the EMT regulator ZEB2," PLoS One, vol. 9, no. 3, article e91827, 2014.

[51] Y. B. Zheng, H. P. Luo, Q. Shi et al., "miR-132 inhibits colorectal cancer invasion and metastasis via directly targeting ZEB2," World Journal of Gastroenterology, vol. 20, no. 21, pp. 65156522, 2014.

[52] K. Kurokawa, T. Tanahashi, T. Iima et al., "Role of miR-19b and its target mRNAs in 5-fluorouracil resistance in colon cancer cells," Journal of Gastroenterology, vol. 47, no. 8, pp. 883-895, 2012.

[53] Z. Jia, K. Wang, A. Zhang et al., "miR-19a and miR-19b overexpression in gliomas," Pathology Oncology Research, vol. 19, no. 4, pp. 847-853, 2013. 
[54] Y. Fan, S. Yin, Y. Hao et al., "miR-19b promotes tumor growth and metastasis via targeting TP53," RNA, vol. 20, no. 6, pp. 765-772, 2014.

[55] M. Liu, R. Yang, U. Urrehman et al., "MiR-19b suppresses PTPRG to promote breast tumorigenesis," Oncotarget, vol. 7, no. 39, pp. 64100-64108, 2016. 


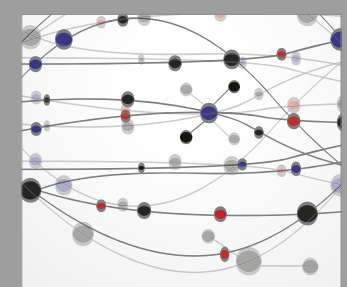

The Scientific World Journal
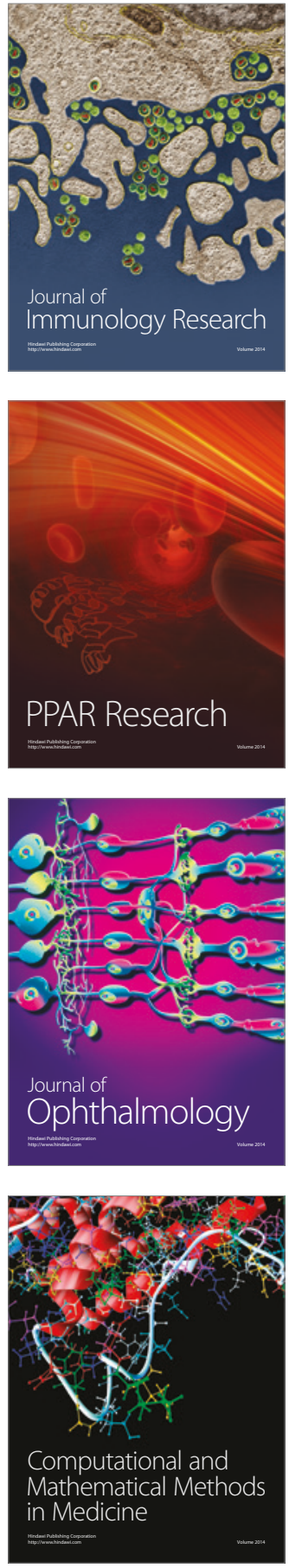

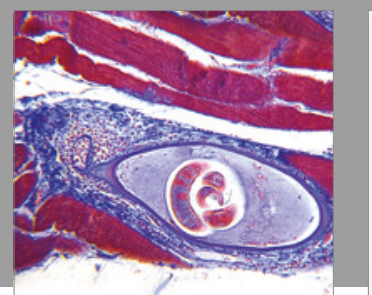

Gastroenterology Research and Practice
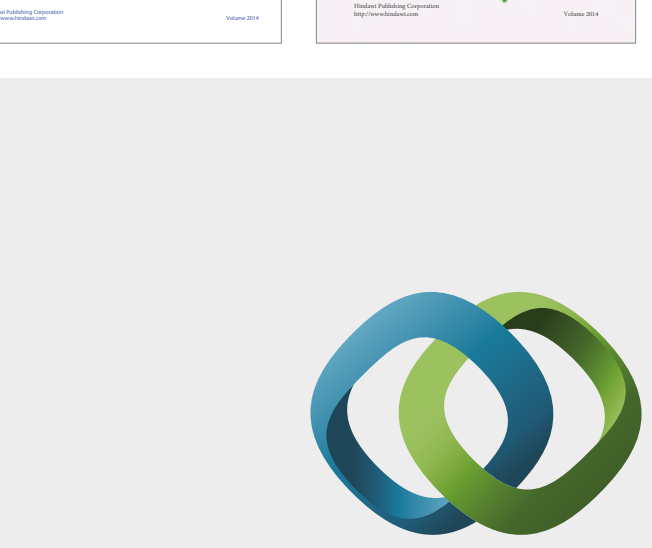

\section{Hindawi}

Submit your manuscripts at

https://www.hindawi.com
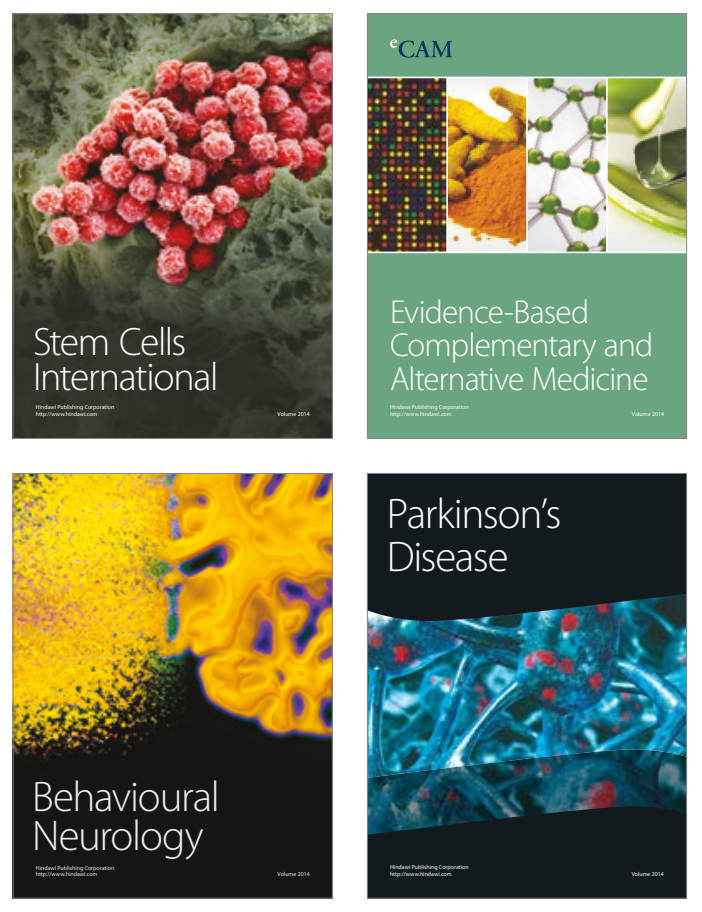
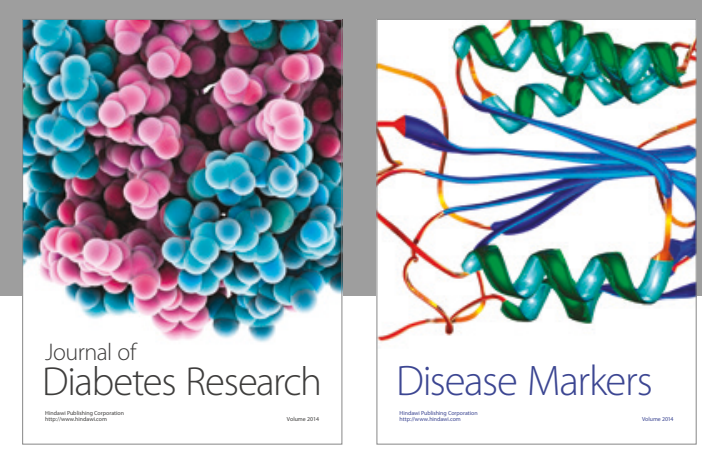

Disease Markers
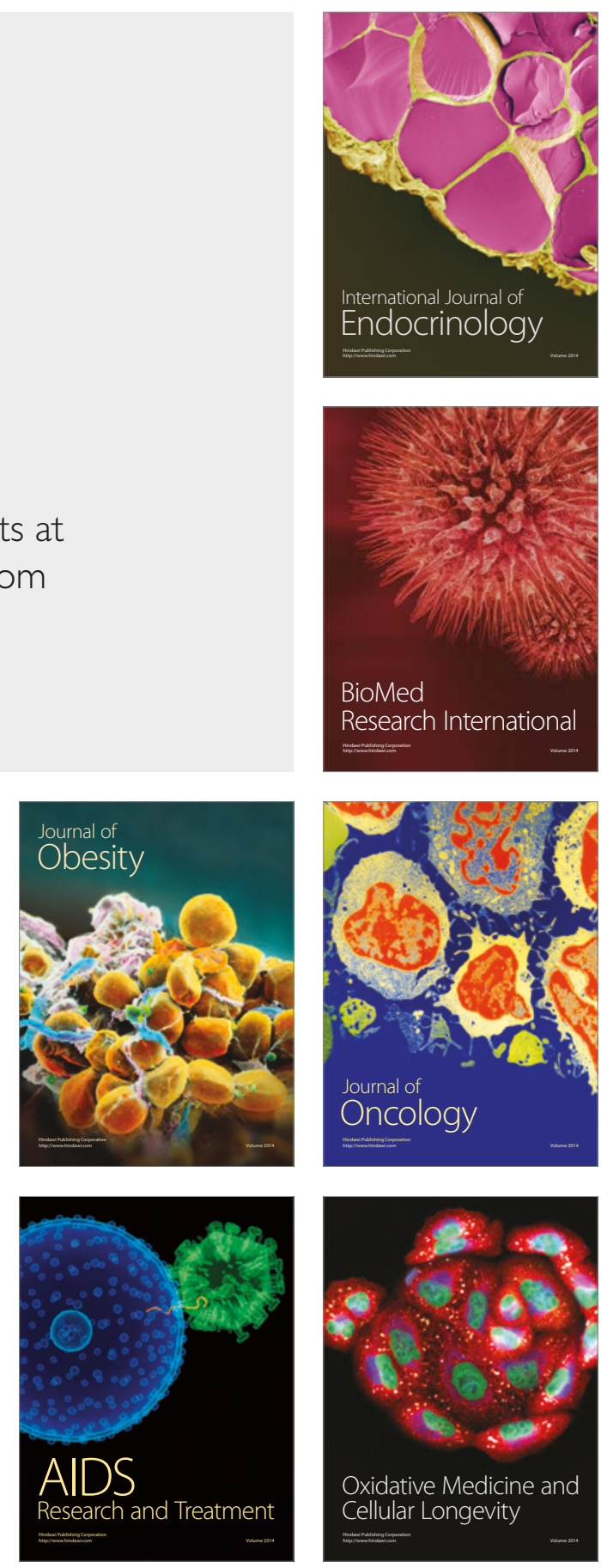